\title{
A review on periodontitis versus endodontics
}

\author{
Mohammad Salarpour ${ }^{1}$, Mahsa Bamadi $^{2}$, Elaheh Salarpour ${ }^{3}$, Masood Bamadi $^{*}$ \\ ${ }^{1}$ Department of Endodontics, School of Dentistry, Zahedan University of Medical Sciences, Zahedan, Iran \\ ${ }^{2}$ International Campus, Zahedan University of Medical Sciences, Zahedan, Iran \\ ${ }^{3}$ School of Dentistry, Zahedan University of Medical Sciences, Zahedan, Iran \\ ${ }^{4}$ Department of Preiodontology, School of Dentistry, Zahedan University of Medical Sciences, Zahedan, Iran; \\ *Corresponding Author: bamadim@yahoo.com
}

Received 19 August 2013; revised 19 September 2013; accepted 19 October 2013

Copyright (C) 2013 Mohammad Salarpour et al. This is an open access article distributed under the Creative Commons Attribution License, which permits unrestricted use, distribution, and reproduction in any medium, provided the original work is properly cited.

\begin{abstract}
Periodontitis (pair-e-o-don-TI-tis) may be a serious gum infection that destroys the soft tissue and bone that support your teeth and endodontics is focused on the detection and treatment of pulp diseases as well. Disease will cause tooth loss or worse, associate with nursing inflated risk of heart failure or stroke and different serious health issues. Endodontics or periodontitis is however common for the most parts preventable. Disease is sometimes the result of poor oral hygiene. Daily brushing and flossing and regular skilled dental cleanings will greatly cut back your probability of developing disease. The purpose of the current review is to explore the important role for periodontitis/endodontics prevention in oral hygiene and health as well. In this review periodontitis/endodontics will be discussed in details in all aspects.
\end{abstract}

Keywords: Periodontitis; Health; Hygiene; Heart Attack; Stroke

\section{PERIODONTITIS}

Periodontitis pron.: /perioudpn'tartis/ or pyorrhea /parə'ria/ could be a set of inflammatory diseases touching the periodontium, i.e., the tissues that surround and support the teeth. Disease involving progressive loss of the alveolar bone round the teeth, and if left untreated, will cause the loosening and future loss of teeth. Disease is caused by microorganisms that adhere to and grow on the tooth's surfaces, in conjunction with a very aggressive response against these microorganisms. An identification of disease is established by inspecting the soft gum tissues round the teeth with a groundwork (i.e., a clinical examination) and by evaluating the patient's
X-ray films (i.e. a picture taking examination), to see the quantity of bone loss round the teeth [1-10]. For specialists within the treatment of disease area unit periodontists; their field is thought as "periodontology" or "periodontics".

The word "periodontitis" comes from the Greek peri, "around", odous (genitive odontos), "tooth", and therefore, the suffix-itis, in medical word, is "inflammation".

\section{CLASSIFICATION}

The 1999 arrangement for dentistry diseases and conditions listed eight major classes of dentistry diseases, [2, $10-18]$ of that $2-6$ area unit termed damaging disease as a result of the harm is basically irreversible. The eight classes area unit as follows:

1) Gingivitis;

2) Chronic disease;

3) Aggressive disease;

4) Periodontal \{disease|disease\} as a manifestation of general disease;

5) Necrotizing lesion gingivitis/periodontitis;

6) Abscesses of the periodontium;

7) Combined periodontic-endodontic lesions.

Moreover, nomenclature expressing each the extent and severity of dentistry diseases area unit appended to the terms higher than to denote the precise diagnosing of a selected patient or cluster of patients.

\section{EXTENT}

The "extent" of illness refers to the proportion of the dentition littered with the illness in terms of share of websites. Sites area unit outlined because the positions at that searching measurements area unit taken around every tooth and, generally, six searching sites around every tooth area unit recorded, as follows:

1) Mesiobuccal; 
2) Midbuccal;

3) Distobuccal;

4) Mesiolingual;

5) Midlingual;

6) Distolingual;

If up to half-hour of websites within the mouth area unit affected, the manifestation is classification as "localized"; for quite half-hour, the term "generalized" is employed [18-20].

\section{SEVERITY}

- The "severity" of unwellness refers to the quantity of dentistry ligament fibers that are lost, termed "clinical attachment loss". Consistent with the yank Academy of Periodontology, the classification of severity is as follows: [3]

- Mild: 1 - 2 mm (0.039-0.079 in) of attachment loss;

- Moderate: 3 - $4 \mathrm{~mm}(0.12-0.16$ in) of attachment loss;

- Severe: $\geq 5 \mathrm{~mm}$ (0.20 in) of attachment loss.

\section{SIGNS AND SYMPTOMS}

In the early stages, periodontitis has very few symptoms; and in many individuals the disease has progressed significantly before they seek treatment.

Symptoms may include:

- Redness or bleeding of gums while brushing teeth, using dental floss or biting into hard food (e.g. apples) (though this may occur even in gingivitis, where there is no attachment loss);

- Gum swelling that recurs;

- Spitting out blood after brushing teeth;

- Halitosis, or bad breath, and a persistent metallic taste in the mouth;

- Gingival recession, resulting in apparent lengthening of teeth. (This may also be caused by heavy-handed brushing or with a stiff tooth brush);

- Deep pockets between the teeth and the gums (pockets are sites where the attachment has been gradually destroyed by collagen-destroying enzymes, known as collagenases);

- Loose teeth, in the later stages (though this may occur for other reasons, as well).

Patients should realize gingival inflammation and bone destruction are largely painless. Hence, people may wrongly assume painless bleeding after teeth cleaning is insignificant, although this may be a symptom of progressing periodontitis in that patient [15-19].

\section{EFFECTS OUTSIDE THE MOUTH}

Periodontitis has been linked to increased inflammation in the body, such as indicated by raised levels of C-reactive protein and interleukin-6 [4-7]. It is linked through this to increased risk of stroke, $[8,9]$ myocardial infarction, [10] and atherosclerosis [11-17]. It also linked in those over 60 years of age to impairments in delayed memory and calculation abilities [18,19]. Individuals with impaired fasting glucose and diabetes mellitus have higher degrees of periodontal inflammation, and often have difficulties with balancing their bloodglucose level owing to the constant systemic inflammatory state, caused by the periodontal inflammation [20,21]. Although no causative connection was proved yet, a recent study revealed an epidemiological association between chronic periodontitis and erectile dysfunction [20-22].

\section{CAUSES}

Periodontitis is AN inflammation of the periodontium, i.e., the tissues that support the teeth. The periodontium consists of 4 tissues:

- gingiva, or gum tissue,

- cementum, or outer layer of the roots of teeth,

- alveolar bone, or the bony sockets into that the teeth ar anchored, and

- periodontal ligaments (PDLs), that ar the animal tissue fibers that run between the cement and also the alveolar bone.

This photographic film displays 2 lone-standing jaw teeth, the lower left 1st bicuspid and canine, exhibiting severe bone loss of $30 \%-50 \%$. Widening of the odontology ligament close the bicuspid is attributable to secondary occlusal trauma.

The primary etiology (cause) of periodontitis is poor or ineffective oral hygiene. That ends up in the buildup of a mycotic [23-26] and microorganism matrix at the gum line, known as plaque. Different contributors ar poor nutrition and underlying medical problems like polygenic disorder [27-35]. New finger nick tests are approved by the Food and Drug Administration within the America, and ar getting used in dental offices to spot and screen patients for potential conducive causes of gum malady, like polygenic disorder.

In some folks, periodontitis progresses to diseasewith the destruction of the animal tissue fibers, the gum tissues become independent from the tooth and gathered fissure, known as an odontology pocket. Subgingival micro-organisms (those that exist beneath the gum line) colonize the odontology pockets and cause additional inflammation within the gum tissues and progressive bone loss. Samples of secondary etiology ar those things that, by definition, cause microorganism plaque accumulation, like restoration overhangs and root proximity.

Smoking is another issue that will increase the incidence of disease, directly or indirectly, [28-30] and will interfere with or adversely have an effect on its treatment [31-33].

Ehlers-Danlos syndrome could be a disease risk issue 
so is that the Papillon-Lefèvre syndrome conjointly referred to as palmoplantar keratodermia.

If left undisturbed, microorganism plaque calcifies to create calculus, that is usually known as tartar. Calculus on top of and below the gum line should be removed fully by the skilled worker or medical man to treat periodontitis and disease. Though the first reason behind each periodontitis and disease is that the microorganism plaque that adheres to the tooth surfaces, there are several different modifying factors. A really sturdy risk issue is one's genetic condition. Many conditions and diseases, together with congenital abnormality, diabetes, and different diseases that have an effect on one's resistance to infection, conjointly increase condition to disease.

Another issue that produces \{periodontitis|periodontal malady|disease \} a tough disease to check is that human host response may have an effect on the alveolar bone biological process. Host response to the bacterial-mycotic insult is especially determined by genetics; but, immune development could play some role in condition.

According to some researchers disease is also related to higher stress [34].

\section{PREVENTION}

Daily oral hygiene measures to forestall periodontitis include:

- Brushing properly on an everyday basis (at least doubly daily), with the patient making an attempt to direct the toothbrush bristles beneath the gum-line, helps disrupt the bacterial-mycotic growth and formation of subgingival plaque.

- Flossing daily and exploitation interdental brushes (if the area between teeth is giant enough), furthermore as cleansing behind the last tooth, the third molar, in every quarter

- Using associate antiseptic gargle: antiseptic gluconate-based mouthwash together with careful oral hygiene could cure periodontal disease, though they can't reverse any attachment loss owing to disease.

- Using odontology trays to keep up dentist-prescribed medications at the supply of the disease: the utilization of trays permits the medication to remain in situ long enough to penetrate the biofilms wherever the micro-organism are found.

- Regular dental check-ups and skilled teeth cleansing as required: Dental check-ups serve to observe the person's oral hygiene ways and levels of attachment around teeth, establish any early signs of disease, and monitor response to treatment.

Typically, dental hygienists (or dentists) use special instruments to scrub (debride) teeth below the gumline and disrupt any plaque growing below the gumline. This can be a customary treatment to forestall from now on progress of established disease. Studies show that once such an expert cleansing (periodontal debridement), microorganism plaque tends to grow back to precleaning levels once regarding 3 to four months. Nevertheless, the continuing stabilization of a patient's odontology state depends for the most part, if not primarily, on the patient's oral hygiene reception, furthermore as on the go. While not daily oral hygiene, periodontitis won't be overcome, particularly if the patient contains a history of in depth periodontitis.

Periodontal disease associated tooth loss are related to a redoubled risk, in male patients, of cancer [36].

\subsection{Initial Therapy}

Removal of microbial plaque and calculus is necessary to establish periodontal health. The first step in the treatment of periodontitis involves nonsurgical cleaning below the gumline with a procedure called scaling and debridement. In the past, root planing was used (removal of cemental layer as well as calculus). This procedure involves use of specialized curettes to mechanically remove plaque and calculus from below the gumline, and may require multiple visits and local anesthesia to adequately complete. In addition to initial scaling and root planing, it may also be necessary to adjust the occlusion (bite) to prevent excessive force on teeth that have reduced bone support. Also, it may be necessary to complete any other dental needs, such as replacement of rough, plaque-retentive restorations, closure of open contacts between teeth, and any other requirements diagnosed at the initial evaluation [30-46].

\subsection{Reevaluation}

Multiple clinical studies have shown nonsurgical scaling and root planing are usually successful if the periodontal pockets are shallower than $4-5 \mathrm{~mm}(0.16-0.20$ in) [37-39]. The dentist or hygienist must perform a reevaluation four to six weeks after the initial scaling and root planing, to determine if the patient's oral hygiene has improved and inflammation has regressed. Probing should be avoided then, and an analysis by gingival index should determine the presence or absence of inflammation. The monthly reevaluation of periodontal therapy should involve periodontal charting as a better indication of the success of treatment, and to see if other courses of treatment can be identified. Pocket depths of greater than $5-6 \mathrm{~mm}(0.20-0.24 \mathrm{in})$ which remain after initial therapy, with bleeding upon probing, indicate continued active disease and will very likely lead to further bone loss over time. This is especially true in molar tooth sites where furcations (areas between the roots) have been exposed [40-46].

\subsection{Surgery}

If nonsurgical therapy is found to have been unsuc- 
cessful in managing signs of disease activity, periodontal surgery may be needed to stop progressive bone loss and regenerate lost bone where possible. Many surgical approaches are used in treatment of advanced periodontitis, including open flap debridement and osseous surgery, as well as guided tissue regeneration and bone grafting. The goal of periodontal surgery is access for definitive calculus removal and surgical management of bony irregularities which have resulted from the disease process to reduce pockets as much as possible. Long-term studies have shown, in moderate to advanced periodontitis, surgically treated cases often have less further breakdown over time and, when coupled with a regular post-treatment maintenance regimen, are successful in nearly halting tooth loss in nearly $85 \%$ of patients [30-41].

\subsection{Maintenance}

Once successful periodontal treatment has been completed, with or without surgery, an ongoing regimen of "periodontal maintenance" is required. This involves regular checkups and detailed cleanings every three months to prevent repopulation of periodontitis-causing microorganism, and to closely monitor affected teeth so early treatment can be rendered if disease recurs. Usually, periodontal disease exists due to poor plaque control, therefore if the brushing techniques are not modified, a periodontal recurrence is probable.

\subsection{Alternative Treatments}

Periodontitis has an inescapable relationship with subgingival calculus (tartar). The first step in any procedure is to eliminate calculus under the gum line, as it houses destructive anaerobic microorganisms that consume bone, gum and cementum (connective tissue) for food.

Most alternative "at-home" gum disease treatments involve injecting antimicrobial solutions, such as hydrogen peroxide, into periodontal pockets via slender applicators or oral irrigators. This process disrupts anaerobic micro-organism colonies and is effective at reducing infections and inflammation when used daily. A number of other products, functionally equivalent to hydrogen peroxide, are commercially available, but at substantially higher cost. However, such treatments do not address calculus formations, and so are short-lived, as anaerobic microbial colonies quickly regenerate in and around calculus [20,33-37].

Additionally, periodontitis can be treated in a noninvasive manner by means of Periostat, an FDA-approved, orally administered drug that has been shown to reduce bone loss. Its mechanism of action in part involves inhibition of matrix metalloproteinases (such as collagenase), which degrade the extracellular matrix under inflammatory conditions. This ultimately can lead to reduction of aveolar bone loss in patients with periodontal disease (as well as patients without periodontitis).

\subsection{Prognosis}

Dentists and dental hygienists measure periodontal disease using a device called a periodontal probe. This thin "measuring stick" is gently placed into the space between the gums and the teeth, and slipped below the gumline. If the probe can slip more than $3 \mathrm{~mm}(0.12 \mathrm{in})$ below the gumline, the patient is said to have a gingival pocket if no migration of the epithelial attachment has occurred or a periodontal pocket if apical migration has occurred. This is somewhat of a misnomer, as any depth is in essence a pocket, which in turn is defined by its depth, i.e., a 2-mm pocket or a 6-mm pocket. However, pockets are generally accepted as self-cleansable (at home, by the patient, with a toothbrush) if they are $3 \mathrm{~mm}$ or less in depth. This is important because if a pocket is deeper than $3 \mathrm{~mm}$ around the tooth, at-home care will not be sufficient to cleanse the pocket, and professional care should be sought. When the pocket depths reach 6 to 7 $\mathrm{mm}(0.24$ to $0.28 \mathrm{in})$ in depth, the hand instruments and cavitrons used by the dental professionals may not reach deeply enough into the pocket to clean out the microbial plaque that cause gingival inflammation. In such a situation, the bone or the gums around that tooth should be surgically altered or it will always have inflammation which will likely result in more bone loss around that tooth. An additional way to stop the inflammation would be for the patient to receive subgingival antibiotics (such as minocycline) or undergo some form of gingival surgery to access the depths of the pockets and perhaps even change the pocket depths so they become $3 \mathrm{~mm}$ or less in depth and can once again be properly cleaned by the patient at home with his or her toothbrush [44-47].

If patients have 7-mm or deeper pockets around their teeth, then they would likely risk eventual tooth loss over the years. If this periodontal condition is not identified and the patients remain unaware of the progressive nature of the disease, then years later, they may be surprised that some teeth will gradually become loose and may need to be extracted, sometimes due to a severe infection or even pain [44-47].

According to the Sri Lankan tea labourer study, in the absence of any oral hygiene activity, approximately $10 \%$ will suffer from severe periodontal disease with rapid loss of attachment ( $>2 \mathrm{~mm} /$ year). About $80 \%$ will suffer from moderate loss ( $1-2 \mathrm{~mm} /$ year $)$ and the remaining $10 \%$ will not suffer any loss $[42,43]$.

\subsection{In Other Animals}

Periodontal disease is the most common disease found in dogs and affects more than $80 \%$ of dogs aged three 
years or older. Its prevalence in dogs increases with age, but decreases with increasing body weight; i.e., toy and miniature breeds are more severely affected. Systemic disease may develop because the gums are very vascular (have a good blood supply). The blood stream carries these anaerobic micro-organisms, and they are filtered out by the kidneys and liver, where they may colonize and create microabscesses. The micro-organisms traveling through the blood may also attach to the heart valves, causing vegetative endocarditis (infected heart valves). Additional diseases that may result from periodontitis include chronic bronchitis and pulmonary fibrosis [46, 47].

\section{ENDODONTICS}

(From the Greek endo "inside"; and odons "tooth") is the dental specialty involved with the study and treatment of the dental pulp. Endodontists perform a range of procedures as well as dentistry medical care (commonly called "root canal therapy"), dentistry retreatment, surgery, treating cracked teeth, and treating dental trauma. Passageway medical care is one in all the foremost common procedures. If the dental pulp (containing nerves, arterioles, venules, animal tissue, and fibrous tissue) becomes unhealthy or lacerate, dentistry treatment is needed to save lots of the tooth. Dentistry is recognized as a specialty by several national dental organizations as well as the yank Dental Association, Royal faculty of Dentists of North American nation, and Royal Australasian faculty of Dental Surgeons.

In the US once finishing a dental degree, a medical practitioner should bear 2 - 3 extra years of postgraduate coaching to become associate degree tooth doctor. Yank Dental Association (CODA) licensed programs area unit a minimum of 2 years long. Following undefeated completion of this coaching the medical practitioner becomes Board eligible to take a seat for the yank Board of Endodontology examination. Undefeated completion of board certification leads to specialist standing within the yank Board of Endodontology [2-18].

There is a unit fifty dentistry coaching programs within the US, during which roughly four hundred students area unit registered. In Iran, the students need at least 4 years of post-graduating programs (master degree).

\section{ENDODONTIC THERAPY OR ROOT CANAL THERAPY}

Colloquially root canal is a sequence of treatment for the pulp of a tooth which results in the elimination of infection and protection of the decontaminated tooth from future microbial invasion. This set of procedures is commonly referred to as a "root canal." Root canals and their associated pulp chamber are the physical hollows within a tooth that is naturally inhibited by nerve tissue, blood vessels and other cellular entities. Endodontic therapy involves the removal of these structures, the subsequent shaping, cleaning, and decontamination of the hollows with tiny files and irrigating solutions, and the obturation (filling) of the decontaminated canals with an inert filling such as gutta percha and typically a eugenolbased cement. Epoxy resin, which may or may not contain Bisphenol A, is employed to bind gutta percha in some root canal procedures [20-27].

After endodontic surgery, the tooth will be "dead," and if an infection is spread at the apex, root end surgery is required.

Although the procedure is relatively painless when done properly, root canal remains a stereotypically fearsome dental operation.

In the situation that a tooth is considered so threatened (because of decay, cracking, etc.) that future infection is considered likely or inevitable, a pulpectomy, removal of the pulp tissue, is advisable to prevent such infection. Usually, inflammation and/or infection is already present within or below the tooth. To cure the infection and save the tooth, the dentist drills into the pulp chamber and removes the infected pulp and then drills the nerve out of the root canal(s) with long needle-shaped hand instrument known as files (H files and $\mathrm{K}$ files) instead. Starting with 15 number $\mathrm{H}$ which is also called as pathfinder and after that files number increased up to 35 in molars and up to $50 / 55$ number file in anterior tooth to broad the canals for a successful treatment.After this is done, the dentist fills each of the root canals and the chamber with an inert material and seals up the opening. This procedure is known as root canal therapy. With the removal of nerves and blood supply from the tooth, it is best that the tooth can be fitted with a crown.

The standard filling material is gutta-percha, a natural polymer prepared from latex from the percha (Palaquium gutta) tree. The standard endodontic technique involves inserting a gutta-percha cone (a "point") into the cleanedout root canal along with cement and a sealer. [5] Another technique uses melted or heat-softened gutta-percha which is then injected or pressed into the root canal passage(s). However, as gutta-percha shrinks as it cools, thermal techniques can be unreliable and sometimes a combination of techniques is used. Gutta-percha is radiopaque, allowing verification afterwards that the root canal passages have been completely filled in, without voids.

An alternative filling material was invented in the early 1950s by Angelo Sargenti. It has undergone several formulations over the years (N2, N2 Universal, RC-2B, $\mathrm{RC}-2 \mathrm{~B}$ White), but all contain paraformaldehyde. The paraformaldehyde, when placed into the root canal, 
forms formaldehyde, which penetrates and sterilizes the passage. The formaldehyde is then theoretically transformed to harmless water and carbon dioxide. The outcome is better than a root canal done with gutta percha according to some investigations. There is however a lack of indisputable, scientifically made studies according to the Swedish Council on Health Technology Assessment.

In the last ten to twenty years, there have been great innovations in the art and science of root canal therapy. Dentists now must be educated on the current concepts in order to optimally perform a root canal. Root canal therapy has become more automated and can be performed faster, thanks to advances in automated mechanical instrumentation of teeth and more advanced root canal filling methods. Most root canal procedures are done in one dental visit, lasting around 1 - 2 hours. Dentists also possess newer technologies that allow more efficient, scientific measurements to be taken of the dimensions of the root canal that must be filled. Many dentists use dental loupes to perform root canals, and the consensus is that root canals performed using loupes or other forms of magnification are more likely to succeed than those performed without them. Although general dentists are becoming versed in these advanced technologies, they are still more likely to be used by specialist root canal doctors (known as endodontists).

Laser root canal procedures are a controversial innovation. Lasers may be fast but have not been shown to thoroughly disinfect the whole tooth, [12] and may cause damage [13].

\section{REFERENCES}

[1] Savage, A., Eaton, K.A., Moles, D.R. and Needleman, I. (2009) A systematic review of definitions of periodontitis and methods that have been used to identify this disease. Journal of Clinical Periodontology, 36, 458-467. http://dx.doi.org/10.1111/j.1600-051X.2009.01408.x

[2] Armitage, G.C. (1999) Development of a classification system for periodontal diseases and conditions. Annals of Periodontology, 4, 1-6. http://dx.doi.org/10.1902/annals.1999.4.1.1

[3] Wiebe, C.B. and Putnins, E.E. (2000) The periodontal disease classification system of the American academy of periodontology - An update. Journal of the Canadian Dental Association, 66, 594-597.

[4] D'Aiuto, F. Parkar, M., Andreou, G., Suvan, H., Brett, P.M., Ready, D. and Tonetti, M.S. (2004) Periodontitis and systemic inflammation: Control of the local infection is associated with a reduction in serum inflammatory markers. Journal of Dental Research, 83, 156-160. http://dx.doi.org/10.1177/154405910408300214

[5] Nibali, L., D’Aiuto, F., Griffiths, G., Patel, K., Suvan, J. and Tonetti, M.S. (2007) Severe periodontitis is associated with systemic inflammation and a dysmetabolic sta- tus: A case-control study. Journal of Clinical Periodontology, 34, 931-937.

http://dx.doi.org/10.1111/j.1600-051X.2007.01133.x

[6] Paraskevas, S., Huizinga, J.D. and Loos, B.G. (2008) A systematic review and meta-analyses on C-reactive protein in relation to periodontitis. Journal of Clinical Periodontology, 35, 277-290. http://dx.doi.org/10.1111/j.1600-051X.2007.01173.x

[7] D'Aiuto, F., Ready, D. and Tonetti, M.S. (2004) Periodontal disease and C-reactive protein-associated cardiovascular risk. Journal of Endodontal Research, 39, 236241.

[8] Pussinen, P.J., Alfthan, G., Jousilahti, P., Paju, S. and Tuomilehto, J. (2007) Systemic exposure to Porphyromonas gingivalis predicts incident stroke. Atherosclerosis, 193, 222-228 http://dx.doi.org/10.1016/j.atherosclerosis.2006.06.027

[9] Pussinen, P.J., Alfthan, G., Rissanen, H., Reunanen, A., Asikainen, S. and Knekt, P. (2004) Antibodies to periodontal pathogens and stroke risk. Stroke, 35, 2020-2023. http://dx.doi.org/10.1161/01.STR.0000136148.29490.fe

[10] Pussinen, P.J., Alfthan, G., Tuomilehto, J., Asikainen, S., Jousilahti, P. (2004) High serum antibody levels to Porphyromonas gingivalis predict myocardial infarction. European Journal of Cardiovascular Prevention \& Rehabilitation, 11, 408-411.

[11] Ford, P.J., Gemmell, E., Timms, P., Chan, A., Preston, F.M. and Seymour, G.J. (2007) Anti-P. gingivalis response correlates with atherosclerosis. Journal of Dental Research, 86, 35-40. http://dx.doi.org/10.1177/154405910708600105

[12] Beck, J.D., Eke, P., Heiss, G., Madianos, P., Couper, D., Lin, D.M., Moss, K., Elter, J., et al. (2005) Periodontal disease and coronary heart disease: A reappraisal of the exposure. Circulation, 112, 19-24. http://dx.doi.org/10.1161/CIRCULATIONAHA.104.511998

[13] Scannapieco, F.A., Bush, R.B. and Paju, S. (2003) Associations between periodontal disease and risk for atherosclerosis, cardiovascular disease, and stroke. A systematic review. Annals of Periodontology, 8, 38-53. http://dx.doi.org/10.1902/annals.2003.8.1.38

[14] Wu, T.J., Trevisan, M., Genco, R.J., Dorn, J.P., Falkner, K.L. and Sempos, C.T. (2000) Periodontal disease and risk of cerebrovascular disease: The first national health and nutrition examination survey and its follow-up study. Archives of International Medicine, 160, 2749-2755. http://dx.doi.org/10.1001/archinte.160.18.2749

[15] Beck, J.D., Elter, J.R., Heiss, G., Couper, D., Mauriello, S.M. and Offenbacher, S. (2001) Relationship of periodontal disease to carotid artery intima-media wall thickness: The Atherosclerosis Risk in Communities (ARIC) study. Arteriosclerosis, Thrombosis, and Vascular Biology, 21, 1816-1822. http://dx.doi.org/10.1161/hq1101.097803

[16] Elter, J.R., Champagne, C.M.E., Beck, J.D. and Offenbacher, S. (2004) Relationship of periodontal disease and tooth loss to prevalence of coronary heart disease. Journal of Periodontology, 75, 782-790. http://dx.doi.org/10.1902/jop.2004.75.6.782

[17] Humphrey, L.L., Fu, R.W., Buckley, D.I., Freeman, M. 
and Helfand, M. (2008) Periodontal disease and coronary heart disease incidence: A systematic review and metaanalysis. Journal of General Internal Medicine, 23, 20792086. http://dx.doi.org/10.1007/s11606-008-0787-6

[18] Noble, J.M., Borrell, L.N., Papapanou, P.N., Elkind, M.S., Scarmeas, N. and Wright, C.B. (2009) Periodontitis is associated with cognitive impairment among older adults: Analysis of NHANES-III. Journal of Neurology, Neurosurgery \& Psychiatry, 80, 1206-1211. http://dx.doi.org/10.1136/jnnp.2009.174029

[19] Kaye, E.K., Valencia, A., Baba, N., Spiro III, A., Dietrich, T. and Garcia, R.I. (2010) Tooth loss and periodontal disease predict poor cognitive function in older men. Journal of the American Geriatrics Society, 58, 713-718. http://dx.doi.org/10.1111/j.1532-5415.2010.02788.x

[20] Zadik, Y., Bechor, R., Galor, S. and Levin, L. (2010) Periodontal disease might be associated even with impaired fasting glucose. British Dental Journal, 208, e20. http://dx.doi.org/10.1038/sj.bdj.2010.291

[21] Soskolne, W.A. and Klinger, A. (2001) The relationship between periodontal diseases and diabetes: An overview. Annals of Edndodontistology, 6, 91-98.

[22] Zadik, Y., Bechor, R., Galor, S., Justo, D. and Heruti, R.J. (2009) Erectile dysfunction might be associated with chronic periodontal disease: Two ends of the cardiovascular spectrum. The Journal of Sexual Medicine, 6, 1111-1116. http://dx.doi.org/10.1111/j.1743-6109.2008.01141.x

[23] Crich, A. (1932) Blastomycosis of the gingiva and jaw. Canadian Medical Association Journal, 26, 662-665.

[24] Urzúa, B., Hermosilla, G., Gamonal, J., Morales-Bozo, I., Canals, M., Barahona, S., Cóccola, C. and Cifuentes, V. (2008) Yeast diversity in the oral microbiota of subjects with periodontitis: Candida albicans and Candida dubliniensis colonize the periodontal pockets. Medical Mycology, 46, 783-793.

http://dx.doi.org/10.1080/13693780802060899

[25] Matsuo, T., Nakagawa, H., Matsuo, N. and Matsuo, N. (1995) Endogenous Aspergillus endophthalmitis associated with periodontitis. Ophthalmologica, 209, 109-111. http://dx.doi.org/10.1159/000310592

[26] Migliari, D.A., Sugaya, N.N., Mimura, M.A. and Cucé, L.C. (1998) Periodontal aspects of the juvenile form of paracoccidioidomycosis. Revista do Instituto de Medicina Tropical de São Paulo, 40, 15-18. http://dx.doi.org/10.1590/S0036-46651998000100004

[27] Lalla, E., Cheng, B., Lal, S., Kaplan, S., Softness, B., Greenberg, E., Goland, R.S. and Lamster, I.B. (2007) Diabetes mellitus promotes periodontal destruction in children. Journal of Clinical Periodontology, 34, 294-298. http://dx.doi.org/10.1111/j.1600-051X.2007.01054.x

[28] Obeid, P. and Bercy, P. (2000) Effects of smoking on periodontal health: A review. Advances in Therapy, 17, 230-237. http://dx.doi.org/10.1007/BF02853162

[29] Tomar, S.L. and Asma, S. (2000) Smoking-attributable periodontitis in the United States: Findings from NHANES III. Journal of Periodontology, 71, 743-751. http://dx.doi.org/10.1902/jop.2000.71.5.743

[30] Ryder, M.I. (2007) The influence of smoking on host responses in periodontal infections. Periodontology, 43 , 267-277.

http://dx.doi.org/10.1111/j.1600-0757.2006.00163.x

[31] Pauletto, N.C., Liede, K., Nieminen, A., Larjava, H. and Uitto, V.-J. (2000) Effect of cigarette smoking on oral elastase activity in adult periodontitis patients. Journal of Periodontology, 71, 58-62. http://dx.doi.org/10.1902/jop.2000.71.1.58

[32] Persson, L., Bergström, J. and Gustafsson, A. (2003) Effect of tobacco smoking on neutrophil activity following periodontal surgery. Journal of Periodontology, 74, 14751482. http://dx.doi.org/10.1902/jop.2003.74.10.1475

[33] Bergström, J. and Boström, L. (2001) Tobacco smoking and periodontal hemorrhagic responsiveness. Journal of Clinical Periodontology, 28, 680-685.

http://dx.doi.org/10.1034/j.1600-051x.2001.028007680.x

[34] Peruzzo, D.C., Benatti, B.B., Ambrosano, G.M.B., Nogueira-Filho, G.R., Sallum, E.A., Casati, M.Z. and Nociti Jr., F.H. (2007) A systematic review of stress and psychological factors as possible risk factors for periodontal disease. Journal of Periodontology, 78, 1491-1504. http://dx.doi.org/10.1902/jop.2007.060371

[35] Michaud, D.S., Liu, Y., Meyer, M., Giovannucci, E. and Joshipura, K. (2008) Periodontal disease, tooth loss, and cancer risk in male health professionals: A prospective cohort study. The Lancet Oncology, 9, 550-558. http://dx.doi.org/10.1016/S1470-2045(08)70106-2

[36] First Evidence Found of Link between Gum Disease and High Alcohol Consumption, Low Dietary Antioxidants.

[37] Stambaugh, R.V., Dragoo, M., Smith, D.M. and Carasali, L. (1981) The limits of subgingival scaling. International Journal of Periodontics \& Restorative Dentistry, 1, 3041.

[38] Waerhaug, J. (1978) Healing of the dento-epithelial junction following subgingival plaque control. I. As observed in human biopsy material. Journal of Periodontology, 49, 1-8. http://dx.doi.org/10.1902/jop.1978.49.1.1

[39] Waerhaug, J. (1978) Healing of the dento-epithelial junction following subgingival plaque control. II: As observed on extracted teeth. Journal of Periodontology, 49, 119134. http://dx.doi.org/10.1902/jop.1978.49.3.119

[40] Kaldahl, W.B., Kalkwarf, K.L., Patil, K.D., Molvar, M.P. and Dyer, J.K. (1996) Long-term evaluation of periodontal therapy: II. Incidence of sites breaking down. Journal of Periodontology, 67, 103-108.

http://dx.doi.org/10.1902/jop.1996.67.2.103

[41] Hirschfeld, L. and Wasserman, B. (1978) A long-term survey of tooth loss in 600 treated periodontal patients. Journal of Periodontology, 49, 225-237. http://dx.doi.org/10.1902/jop.1978.49.5.225

[42] Preus, H.R., Anerud, A., Boysen, H., Dunford, R.G., Zambon, J.J. and Loe, H. (1995) The natural history of periodontal disease. The correlation of selected microbiological parameters with disease severity in Sri Lankan tea workers. Journal of Clinical Endodontics, 22, 674-678.

[43] Ekanayaka, A. (1984) Tooth mortality in plantation workers and residents in Sri Lanka. Community Dentistry and Oral Epidemiology, 12, 128-135. 
http://dx.doi.org/10.1111/j.1600-0528.1984.tb01425.x

[44] WHO (2002) Mortality and burden of disease estimates for WHO member states in 2002 (xls). World Health Organization.

[45] Vos, T. (2012) Years lived with disability (YLDs) for 1160 sequelae of 289 diseases and injuries 1990-2010: A systematic analysis for the global burden of disease study 2010. Lancet, 380, 2163-2196.

http://dx.doi.org/10.1016/S0140-6736(12)61729-2
[46] Zadik, Y., Bechor, R., Shochat, Z. and Galor, S. (2008) Ethnic origin and alveolar bone loss in Israeli adults. Refuat Hapeh Vehashinayim, 25, 19-22,72.

[47] Muller-Esnault, S.D.V.M. (2009) Endodontal disease in the dog and cat.

http://www.critterology.com/articles/periodontal-disease$\underline{\text { dog-and-cat }}$ 